\title{
Scaling Trapped Ion Quantum Computers Using Fast Gates and Microtraps
}

\author{
Alexander K. Ratcliffe, ${ }^{*}$ Richard L. Taylor, and Joseph J. Hope \\ Department of Quantum Science, RSPE, Australian National University, \\ Canberra, Australian Capital Territory 2601, Australia \\ André R. R. Carvalho \\ Centre for Quantum Dynamics, Griffith University, Gold Coast, Queensland 4222, Australia
}

(Received 16 November 2017; published 29 May 2018)

\begin{abstract}
Most attempts to produce a scalable quantum information processing platform based on ion traps have focused on the shuttling of ions in segmented traps. We show that an architecture based on an array of microtraps with fast gates will outperform architectures based on ion shuttling. This system requires higher power lasers but does not require the manipulation of potentials or shuttling of ions. This improves optical access, reduces the complexity of the trap, and reduces the number of conductive surfaces close to the ions. The use of fast gates also removes limitations on the gate time. Error rates of $10^{-5}$ are shown to be possible with $250 \mathrm{~mW}$ laser power and a trap separation of $100 \mu \mathrm{m}$. The performance of the gates is shown to be robust to the limitations in the laser repetition rate and the presence of many ions in the trap array.
\end{abstract}

DOI: 10.1103/PhysRevLett.120.220501

The promise of quantum devices to benefit modern computing technology, such as the simulation of quantum systems and new encryption technologies, relies on the scaling properties of quantum information processing compared to classical computing [1]. Thus far, no platform has achieved a scale that outperforms classical computers. Architectures based on trapping ions in a single linear trap have achieved all the operations required for viable quantum information processing [2-10]; however, like all other platforms, they are currently limited in the scalability of the number of qubits [11].

While many proposed quantum computing platforms can generate large numbers of qubits, the key figure of merit is the number of high-fidelity entangling operations performable over their decoherence time. The speed of the gate operation therefore causes a bound on scalability. Putting more ions in linear Paul traps, for example, requires the trapping frequency to be lowered to prevent buckling of the ion chain [11]. This increases the time for sidebandresolving adiabatic gates, which operate slower than the trapping period [12]. Attempts to overcome this limitation have focused on several schemes using 2D arrays, where ions are moved closer when performing gate operations $[13,14]$, confined in Wigner crystals inside Penning traps $[15,16]$, or segmented linear Paul traps, where ions are moved between traps [17-22]. The fastest demonstrated shuttling processes, which use schemes to relax the adiabatic criteria $[23,24]$, have been of the order of 5 trap periods. While it may be possible to push the limits of adiabaticity further, until ions are moved within one or two trap periods [25], this still limits the number of gate operations achievable before the state decoheres.
Nonadiabatic gates ("fast gates") were proposed [26,27] to overcome limitations on the gate time posed by sideband-resolving adiabatic gates. Rather than attempting to resolve motional sidebands, fast gate schemes use broadband pulse sequences to entangle the ions and to restore the ionic motion after the gate operation. Fast gates improve prospects for larger computations in linear Paul traps and have been recently demonstrated experimentally [28-30]. Even with fast gates, practical challenges of scalability in these geometries remain [31-33], with computation in a linear Paul trap scaling to $40-50$ ions before the error due to compounding gates becomes significant [33]. Additionally, addressing individual ions becomes increasingly difficult as their separation decreases [34].

We propose a method of overcoming these limits using fast gates to produce entangling operations between separate microtraps. An architecture based on trapping ions in individual microtraps has previously been proposed [35], which required a separate ion to be shuttled around the array. A more recent proposal specified a simple electrode design for a series of parallel linear traps, where magnetic field gradients could be used to simulate spin-spin interactions for a quantum simulation [36]. This architecture allows for ions to be localized close to the minima of the microtrap potentials; hence, the resolution of ion location is not limited by the number of ions in the trap. The spatial resolution is then determined by the experimental design, and the minimum distance is limited by the feature size of the trap. Fast gates require high laser power but do not require ion shuttling, magnetic field gradients, or timedependent potentials. We show that this proposed architecture using fast gates in microtrap arrays provides greater 
(a)
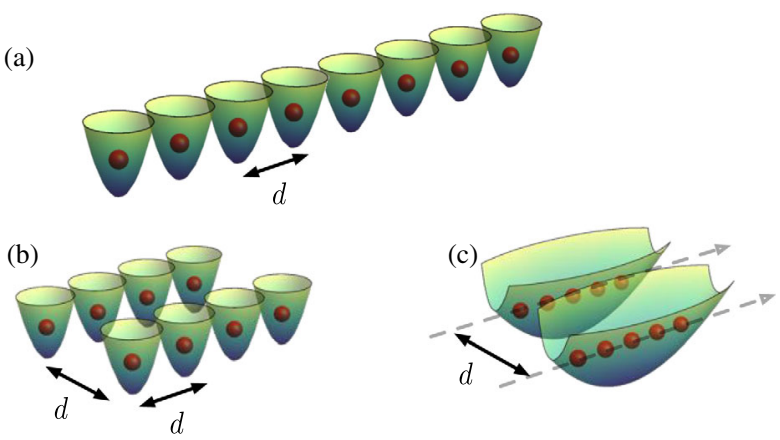

FIG. 1. (a) Diagram with a 1D lattice of trapped ions sitting in individual microtraps separated by a distance $d$. In this Letter, we model this situation. In the future, more scalable arrangements will likely consist of arrays of traps holding single or multiple ions, as shown in (b) and (c). Fast gates can then be used efficiently between nearby ions from different traps, without the need for the potentials to be changed or for the ions to be moved.

scalability than both current ion shuttling-based platforms and fast gate schemes based on linear traps. We show that the laser parameters required to connect ions between adjoining linear traps with high fidelity are achievable.

Multiqubit gates for trapped ions rely on the strong Coulomb interaction they share. When ions are trapped separately, that Coulomb interaction is generally weaker. Rather than attempting to move the ions closer, we find that fast gate schemes can still produce high-fidelity gates in less than a trap period. Consider a set of Paul traps each containing a single ion as shown in Fig. 1(a), arranged in a linear chain with the minima between the nearest neighboring traps separated by some distance $d$. Microtrap designs already exist where $d$ is as small as $100 \mu \mathrm{m}[5,37]$. We examine the feasibility of using fast gates to execute twoqubit gates along this chain and show that, as the length of the ion chain increases, the fidelity does not decay indefinitely. Ultimately, we envisage that these gates will operate between neighboring ions in arrays of individual traps, as shown in Figs. 1(b) and 1(c). The use of Rydberg ions could also be used to enhance this interaction through their dipole and quadrupole coupling [38,39].

Fast gates use laser pulses to apply state-dependent momentum kicks on pairs of ions, inducing state-dependent energy shifts through the Coulomb interaction between the ions. Well-chosen strengths and timing of kicks can create a state-dependent phase shift and simultaneously return the motional state of the ions to their initial state. This creates a controlled phase gate $\hat{U}_{\text {CPhase }}=e^{i(\pi / 4) \sigma_{1}^{2} \sigma_{2}^{2}}$, which is a sufficient entangling gate for universal computation. Different numbers of pulses and ratios between kick strengths define different schemes.

The gate schemes we examine here are a generalization of the fast robust antisymmetric gate (FRAG) scheme [31], a variant of the Garcia-Ripoll, Zoller, and Cirac scheme [26]. They consist of six groups of counterpropagating $\pi$ pulses incident on the ions to be entangled. These pulse groups are defined by fixed ratios of pulses and some global scaling of the pulse number given by the factor $n$. We evaluate the gate fidelity as a function of the experimental design and required total gate time and find that the important elements of the experimental design can be reduced to a single dimensionless parameter. Further details of the model, approximations used, and the FRAG scheme can be found in Supplemental Material [40].

We use numerical searches to find pulse timings that produce high-quality gate operations, with the stateaveraged fidelity $F$, given as the fidelity of the postgate state with the target state integrated over all initial states. This is efficient to compute and strongly related to other distance measures for high-fidelity gates. Within the optimization, we impose an upper bound on the pulse timings, equivalent to setting a maximum gate time. Optimization is then run over a set of increasing upper bounds, which allows for a simple numerical optimization and analysis of the relationship between the gate time and infidelity $1-F$. See Sec. III of Supplemental Material for details of the optimization [40].

It is sensible to report results in terms of $1-F$, because we examine fidelities extremely close to unity. While our numerical calculations use the full infidelity defined above, a Taylor expansion of $1-F$, justified by its small value and given in full in Supplemental Material [40], reveals the important parameters of the problem and helps guide our numerical optimization.

The first important quantity is the normalized difference between the breathing mode frequency $\omega_{\mathrm{BR}}$ and the common motional mode frequency $\omega$ :

$$
\chi=\frac{\omega_{\mathrm{BR}}-\omega}{\omega},
$$

corresponding to the relative spacing of the vibrational mode spectrum. We express this as a function of the experimentally relevant parameter $\xi=\left(d^{3} \omega^{2} / \alpha\right)$ with a simpler form, where $\alpha=\left(e^{2} / 4 \pi \varepsilon_{0}\right)(1 / M)$. Here $e$ is the electron charge, $M$ the mass of the ions, and $\varepsilon_{0}$ the vacuum permittivity. See Sec. IV of Supplemental Material [40] for further details of the derivation of $\chi$ and expression in terms of $\xi$.

For a fixed value of the Lamb-Dicke parameter $\eta$, the infidelity is fully described by the number of pulses in each pulse train and trigonometric expressions of $\chi$. A derivation of this is shown in Supplemental Material [40]. The schemes we consider have either $n$ or $2 n$ pulses in each pulse train, where $n$ is a positive integer. Therefore, $n$ and $\chi$ completely specify the optimal infidelities as a function of the dimensionless gate time $\tau_{G}$ expressed in trap periods $\tau_{G}=\left(\omega t_{G} / 2 \pi\right)$.

To analyze the performance of fast gates in microtraps, we find optimized gates for a large range of values of $n$ and $\chi$, as a function of operational gate time $\tau_{G}$. For some parameter regimes, the optimized fidelity gate takes the maximum gate time allowed by the optimization, but for 


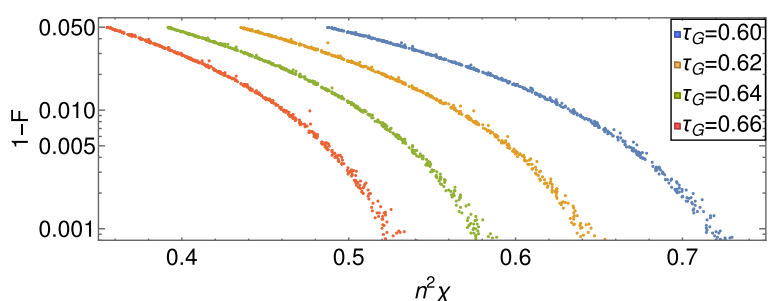

(a)

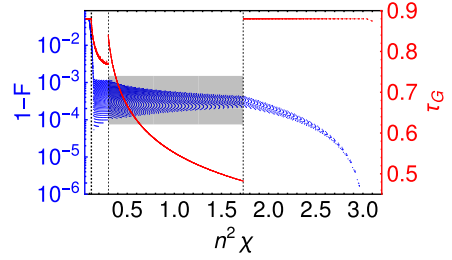

(b)

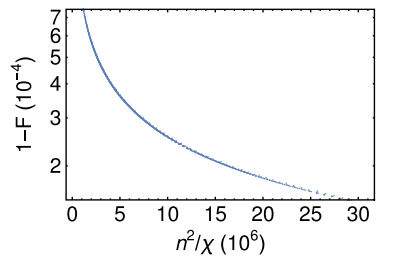

(c)
FIG. 2. The infidelity of a two-ion gate plotted for many different values of $n$ and $\chi$. Despite considerable structure in these solutions, there are two types of regions in parameter space where the gate solutions are locally simple. (a) Infidelity as a function of $n^{2} \chi$ for several gate time caps imposed by the optimization. This shows that $n^{2} \chi$ is a useful single dimensionless parameter for predicting infidelity in this parameter region. (b) Infidelity (blue, left vertical axis) and total gate time (red, right vertical axis) as a function of the single nondimensional parameter $n^{2} \chi$. We see the optimized solution transition from taking the full allowed time to taking less time than allowed, while the infidelity stops being well defined by $n^{2} \chi$. For larger values of $n$, new gate solutions of the first type appear but with much lower infidelities. (c) Infidelity of the gates shown in the gray shaded section in (b), showing that in this region $n^{2} / \chi$ is a good predictor of infidelity.

other parameter regimes the highest-quality solutions are faster. When our optimized solutions take as long as the gate time upper bound, their infidelities bunch along almost monotonic curves when plotted as a function of $n^{2} \chi$, shown in Fig. 2(a). When they take less time than the optimization upper bound, shown in Fig. 2(b), their infidelities are an almost monotonic function of $n^{2} / \chi$, as shown in Fig. 2(c). As $n$ increases, the achievable infidelity goes down without limit as new classes of solution are found, requiring increasing laser power. We find that, with a trap separation of $100 \mu \mathrm{m}$, a $1 \mathrm{MHz}$ trap, and a gate time just under the trap period, a laser power of around $100 \mathrm{~mW}$ allows infidelities of $10^{-2}$, which is sufficient to implement fault-tolerant computation using surface codes [41]. Achieving $10^{-5}$ infidelity to implement earlier proposed schemes [42] would require a laser power of $250 \mathrm{~mW}$.

Rather than fixing the maximum gate time and examining the infidelity, we can do the reverse. The distribution of optimized gates with a fidelity greater than $99 \%$ is shown in Fig. 3. The empty regions in this figure indicate parameter choices where the optimized gate requires less time than the maximum allowed, so there is a gap until a higher-fidelity solution exists with a longer gate time. The different schemes caused by the reordering of the kick timings

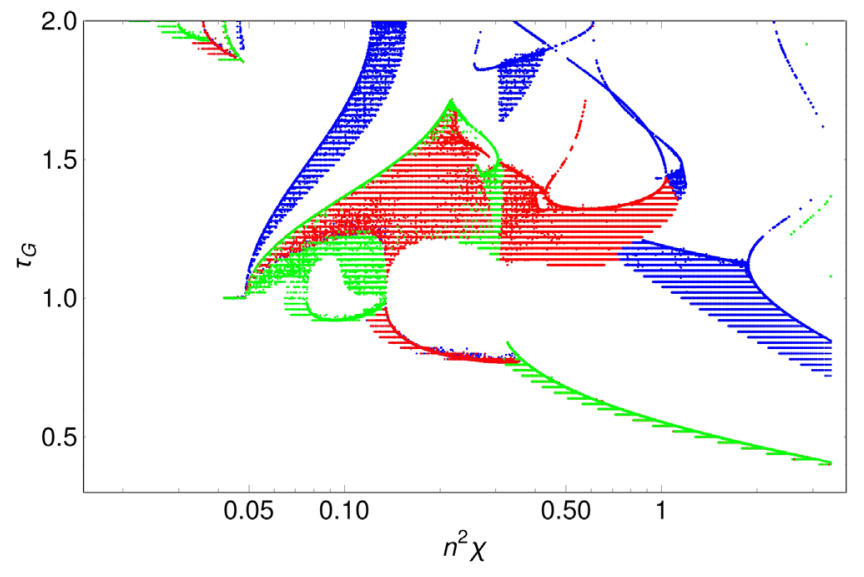

FIG. 3. Gate solutions with infidelities less than $10^{-2}$ as a function of the gate time in trap periods $\tau_{G}$ and the parameter $n^{2} \chi$. Different orderings of pulses and hence different schemes are denoted by different colors. Here the FRAG scheme is indicated by blue, while the red and green represent schemes with different pulse orderings to the FRAG scheme. This shows that the optimal scheme is generally dependent on the choice of the gate time and experimental parameter choice. The empty regions show that sometimes increasing the allowed gate time will not result in decreased gate infidelity unless a threshold gate time is reached, as indicated by the next colored region.

are shown as different colored regions within Fig. 3. We can see that there are distinct regions where one of these schemes provides the optimal gate and that the distinct "jumps" in parameter space are associated with a changing scheme. The changes of behavior in Fig. 2(b) correspond to reaching the edge of an empty region in Fig. 3.

The optimal system for gate performance in both infidelity and nondimensional time will be one that maximizes the value of $n$. Depending on the experimental limitations, it may be advantageous to maximize $\chi$, achieved by decreasing the separation of microtraps or decreasing the trapping frequency. However, decreasing the trapping frequency will also increase the total dimensional gate time. It is thus optimal to only decrease the trap separation to improve gate times. The description of performance using the parameter $n^{2} \chi$ demonstrates that the parameter $n$ has a more significant impact on performance than $\chi$. This indicates that a key focus for improving gate performance should be to increase the number of pulses in a train and, hence, the repetition rate of the laser used.

Thus far, this analysis comes from simulations of pairs of qubits; however, the performance for larger quantities of qubits must be examined. Importantly, we must assess the scaling performance as a function of the parameter $\chi$. Maximizing $n$ is always beneficial in both time and fidelity, but changing $\chi$ and the number of microtraps both affect the dynamics of the ion chain. We use gates optimized for a simple two-ion system and apply these to a system with more ions. This method will not, in general, produce the highest-fidelity gates for systems with more ions, but it 


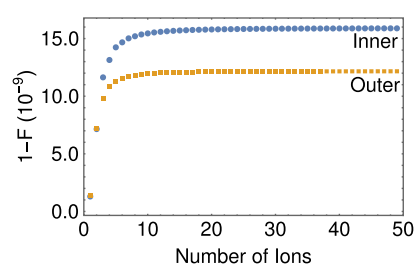

(a)

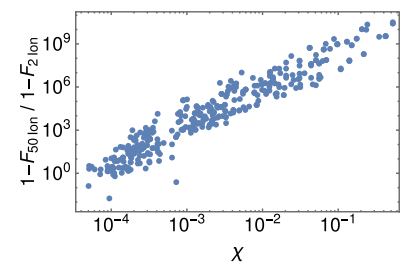

(b)

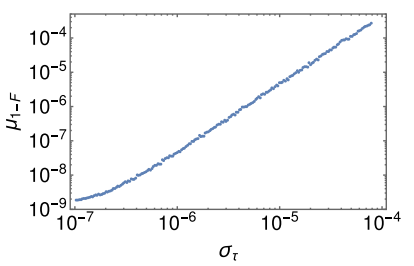

(a)

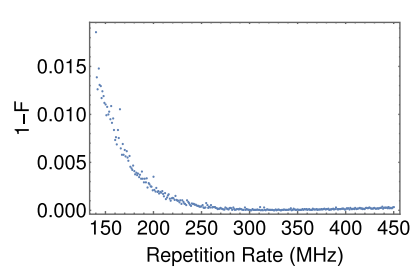

(b)

FIG. 4. (a) Infidelity of a two-ion optimized gate $(n=50$, $\chi=1.8 \times 10^{-4}$, gate time of 1.4 trap periods), with fixed pulse timings and gate time, with an increasing number of microtraps in the processor. Shown for both the innermost pair of ions and the outermost pair of ions. Here the infidelity is for the motional and electronic state of all the ions in the chain. (b) Ratios of the infidelity of a gate applied to a 50-ion array to the infidelity of that gate applied to a two-ion array as a function of $\chi$. Showing microtraps with a low $\chi$ scale more effectively than linear traps when using gates optimized for two-qubit systems.

allows for computationally feasible searches. As a result, the gate time and pulse timings remain fixed over a changing number of ions. The fidelity includes the motional states of all the ions in the chain, and the electronic states of the ions not being operated on are implicitly preserved. Hence, this provides the complete fidelity for the multi-ion system under a gate operation.

Figure 4(a) shows that gate fidelities initially decrease as the number of ions is increased but plateau for chains of around ten ions or more. This is due to the decreasing impact of additional ions to the motional modes of the system. This trend is observed when applying gates between any two adjacent ions in the system. Here we have investigated the impact on gates applied to a pair of ions in the middle and on the edge of the chain, as they exhibit the lowest and highest infidelities, respectively.

Trap geometries also affect the scaling of two-qubit optimized gates. Figure 4(b) shows that there is a clear dependence on $\chi$. The data were created using a set of randomly chosen high-fidelity gates with various values of $\chi$, which were then applied to both a two-ion system and a 50 -ion system. The ratio of the infidelities for these two systems shows a clear linear trend in a log-log plot. Here this indicates a cubic relationship between the ion scaling performance and the parameter $\chi$.

An important feature of any quantum information processing architecture is the performance of the architecture under realistic noise and experimental error. The first error we investigate is encountered in systems using delay loops to achieve multiple coincident pulses, where there is some error on the timing of the pulses. This error was simulated by applying a Gaussian noise with a varying standard deviation to the gate timings. The resulting distribution of infidelities was observed to be exponential; we use the mean of this distribution as a measure of the average infidelity. The impact of this form of noise on the infidelity is significant [see Fig. 5(a)], indicating that heavy

FIG. 5. (a) Mean infidelities of an optimized gate $(n=50$, gate time of 1.4 trap periods) against the standard deviation of the Gaussian noise applied to the timings, given in absolute trap periods. Demonstrating fidelity is significantly decreased when imperfect pulse timings are applied; this result was consistent for different parameters. (b) An optimized gate $(n=24, \omega=2 \pi \mathrm{MHz}$, gate time of $2.5 \mu \mathrm{s}$ ), showing the infidelity for varying finite repetition rates. The minimum repetition rate required to resolve pulse trains is $135 \mathrm{MHz}$.

attention should be paid to ensure correct timings of gate pulses when using a method of delay loops. Accuracies required for high fidelities are a factor of $10^{4}$ shorter than the trapping period.

The alternate option of using a pulse picker to generate pulse trains results in both a spreading of the pulses that make up the impulses $n_{j}$ defined in the FRAG scheme as well as a small shift in the mean timings $\tau_{j}$ of those impulses due to the discrete pulse times. We see that the gate infidelity remains remarkably robust to a finite repetition rate. In Fig. 5(b), we see that gates maintain a high fidelity up to the point where different groups of pulses would be required to overlap. This agrees with results previously obtained for the FRAG gate scheme in linear Paul traps [31]. The nonmonotonicity of the infidelity is a result of aforementioned shift in the mean gate times.

Taking all these limitations into account, we conclude that high-fidelity fast gates can be executed between microtraps using currently available technology. As an example, we find a scheme that is capable of producing a controlled phase gate in 2.5 trap periods with a fidelity of $99.8 \%$ and $99.995 \%$, using repetition rates of 200 and 300 times the trapping frequency, respectively. Using $\mathrm{Ca}^{+}$ions, an $\sim 1 \mathrm{MHz}$ trapping frequency and $100 \mu \mathrm{m}$ trap separation $\left(\chi=1.8 \times 10^{-4}\right)$, as used in some current experimental setups $[37,43]$, correspond to a counterpropagating $\pi$-pulse repetition rate of 200 and $300 \mathrm{MHz}$, respectively. This would result in gate times of $2.5 \mu \mathrm{s}$. These requirements are consistent with the $300 \mathrm{MHz}$ repetition rates, achieved with a laser power of $190 \mathrm{~mW}$ [44]. There is a linear relationship between the $\pi$-pulse repetition rate and laser power; hence, for a $200 \mathrm{MHz}$ repetition rate, a laser power of $130 \mathrm{~mW}$ should be expected. This is a comparable gate time and infidelity to that reported for experimental fast gates in a linear trap [28] but works across separate microtraps, providing significant benefits to scalability.

In conclusion, we propose that a microtrap architecture with fast gates provides an experimentally realizable 
platform for dramatically improved scaling for trapped ion quantum information processing platforms. This architecture compares favorably to current state of the art platforms based on ion shuttling while requiring less complexity in the trap geometry.

It was shown that gate fidelity was surprisingly robust to effects of the finite laser repetition rate for the counterpropagating $\pi$ pulses. The repetition rate need only be sufficient to maintain a separation of pulse trains, which was shown to be experimentally feasible under a standard set of experimental parameters.

It was shown that the fidelities of the fast gates do not decay indefinitely as the number of ions increases. Microtrap architectures also allow multidimensional arrays. This has significant potential performance benefits, as the penalties for scaling in each dimension are independent, allowing considerably better fidelities for a given number of qubits. Furthermore, the increased connectivity between distant ions when using nearest-neighbor interactions helps $2 \mathrm{D}$ or $3 \mathrm{D}$ systems to require many fewer gates to perform any given algorithm.

The authors thank R. Blatt for providing useful comments and providing details on current ion trapping experiments. This work was supported by the Australian Government through the Australian Research Council's Discovery Projects funding scheme (Project No. DP130101613).

*u6141396@anu.edu.au

[1] M. A. Nielsen and I. L. Chuang, Quantum Computation and Quantum Information (Cambridge University Press, Cambridge, England, 2010), p. 702.

[2] D. Leibfried, B. DeMarco, V. Meyer, D. Lucas, M. Barrett, J. Britton, W. M. Itano, B. Jelenković, C. Langer, T. Rosenband, and D. J. Wineland, Nature (London) 422 , 412 (2003).

[3] Y. H. Chen, Y. Xia, Q. Q. Chen, and J. Song, Phys. Rev. A 91, 012325 (2015)

[4] D. Kielpinski, Front. Phys. China 3, 365 (2008).

[5] D. Stick, W. K. Hensinger, S. Olmschenk, M. J. Madsen, K. Schwab, and C. Monroe, Nat. Phys. 2, 36 (2006).

[6] S. Seidelin, J. Chiaverini, R. Reichle, J. J. Bollinger, D. Leibfried, J. Britton, J. H. Wesenberg, R. B. Blakestad, R. J. Epstein, D. B. Hume, W. M. Itano, J. D. Jost, C. Langer, R. Ozeri, N. Shiga, and D. J. Wineland, Phys. Rev. Lett. 96, 253003 (2006).

[7] T. P. Harty, D. T. C. Allcock, C. J. Ballance, L. Guidoni, H. A. Janacek, N. M. Linke, D. N. Stacey, and D. M. Lucas, Phys. Rev. Lett. 113, 220501 (2014).

[8] C. Monroe, D. M. Meekhof, B. E. King, W. M. Itano, and D. J. Wineland, Phys. Rev. Lett. 75, 4714 (1995).

[9] J. Benhelm, G. Kirchmair, C. F. Roos, and R. Blatt, Nat. Phys. 4, 463 (2008).

[10] S. Debnath, N. M. Linke, C. Figgatt, K. A. Landsman, K. Wright, and C. Monroe, Nature (London) 536, 63 (2016).
[11] D. Wineland, C. Monroe, W. Itano, D. Leibfried, B. King, and D. Meekhof, J. Res. Natl. Inst. Stand. Technol. 103, 259 (1998).

[12] F. Schmidt-Kaler, S. Gulde, M. Riebe, T. Deuschle, A. Kreuter, G. Lancaster, C. Becher, J. Eschner, H. Häffner, and R. Blatt, J. Phys. B 36, 623 (2003).

[13] R. B. Blakestad, C. Ospelkaus, A. P. Vandevender, J. M. Amini, J. Britton, D. Leibfried, and D. J. Wineland, Phys. Rev. Lett. 102, 153002 (2009).

[14] H. Häffner, C. F. Roos, and R. Blatt, Phys. Rep. 469, 155 (2008).

[15] J. D. Baltrusch, A. Negretti, J. M. Taylor, and T. Calarco, Phys. Rev. A 83, 042319 (2011).

[16] J. G. Bohnet, B. C. Sawyer, J. W. Britton, M. L. Wall, A. M. Rey, M. Foss-Feig, and J. J. Bollinger, Science 352, 1297 (2016).

[17] D. Kielpinski, C. Monroe, and D. J. Wineland, Nature (London) 417, 709 (2002).

[18] J. M. Amini, H. Uys, J. H. Wesenberg, S. Seidelin, J. Britton, J. J. Bollinger, D. Leibfried, C. Ospelkaus, A. P. Vandevender, and D. J. Wineland, New J. Phys. 12, 033031 (2010).

[19] R. Reichle, D. Leibfried, R. B. Blakestad, J. Britton, J. D. Jost, E. Knill, C. Langer, R. Ozeri, S. Seidelin, and D. J. Wineland, Fortschr. Phys. 54, 666 (2006).

[20] S. Schulz, U. Poschinger, K. Singer, and F. Schmidt-Kaler, Fortschr. Phys. 54, 648 (2006).

[21] G. Huber, T. Deuschle, W. Schnitzler, R. Reichle, K. Singer, and F. Schmidt-Kaler, New J. Phys. 10, 013004 (2008).

[22] H. Kaufmann, T. Ruster, C. T. Schmiegelow, F. SchmidtKaler, and U. G. Poschinger, New J. Phys. 16, 073012 (2014).

[23] E. Torrontegui, S. Ibáñez, S. Martínez-Garaot, M. Modugno, A. del Campo, D. Guéry-Odelin, A. Ruschhaupt, X. Chen, and J. G. Muga, Adv. At. Mol. Opt. Phys. 62, 117 (2013).

[24] A. Walther, F. Ziesel, T. Ruster, S. T. Dawkins, K. Ott, M. Hettrich, K. Singer, F. Schmidt-Kaler, and U. Poschinger, Phys. Rev. Lett. 109, 080501 (2012).

[25] R. Blatt (private communication).

[26] J. J. Garcia-Ripoll, P. Zoller, and J. I. Cirac, Phys. Rev. Lett. 91, 157901 (2003).

[27] L. M. Duan, Phys. Rev. Lett. 93, 100502 (2004).

[28] V. M. Schäfer, C. J. Ballance, K. Thirumalai, L. J. Stephenson, T. G. Ballance, A. M. Steane, and D. M. Lucas, Nature (London) 555, 75 (2018).

[29] J. D. Wong-Campos, S. A. Moses, K. G. Johnson, and C. Monroe, Phys. Rev. Lett. 119, 230501 (2017).

[30] K. G. Johnson, J. D. Wong-Campos, B. Neyenhuis, J. Mizrahi, and C. Monroe, Nat. Commun. 8, 697 (2017).

[31] C. D. B. Bentley, A. R. R. Carvalho, and J. J. Hope, New J. Phys. 17, 103025 (2015).

[32] C. D. B. Bentley, R. L. Taylor, A. R. R. Carvalho, and J. J. Hope, Phys. Rev. A 93, 042342 (2016).

[33] R. L. Taylor, C. D. B. Bentley, J. S. Pedernales, L. Lamata, E. Solano, A. R. R. Carvalho, and J. J. Hope, Sci. Rep. 7, 46197 (2017).

[34] C. Monroe and J. Kim, Science 339, 1164 (2013).

[35] J. I. Cirac and P. Zoller, Nature (London) 404, 579 (2000).

[36] J. Welzel, A. Bautista-Salvador, C. Abarbanel, V. WinemanFisher, C. Wunderlich, R. Folman, and F. Schmidt-Kaler, Eur. Phys. J. D 65, 285 (2011). 
[37] M. Kumph, P. Holz, K. Langer, M. Meraner, M. Niedermayr, M. Brownnutt, and R. Blatt, New J. Phys. 18, 023047 (2016).

[38] G. Higgins, F. Pokorny, C. Zhang, Q. Bodart, and M. Hennrich, Phys. Rev. Lett. 119, 220501 (2017).

[39] M. Müller, L. Liang, I. Lesanovsky, and P. Zoller, New J. Phys. 10, 093009 (2008).

[40] See Supplemental Material at http://link.aps.org/ supplemental/10.1103/PhysRevLett.120.220501, for the detailed description of the model, gate schemes, optimization, and nondimensional parameter derivation.
[41] A. G. Fowler, M. Mariantoni, J. M. Martinis, and A. N. Cleland, Phys. Rev. A 86, 032324 (2012).

[42] A. M. Steane, Nature (London) 399, 124 (1999).

[43] M. Niedermayr, K. Lakhmanskiy, M. Kumph, S. Partel, J. Edlinger, M. Brownnutt, and R. Blatt, New J. Phys. 16, 113068 (2014).

[44] M. I. Hussain, M. J. Petrasiunas, C. D. B. Bentley, R. L. Taylor, A. R. R. Carvalho, J. J. Hope, E. W. Streed, M. Lobino, and D. Kielpinski, Opt. Express 24, 16638 (2016). 